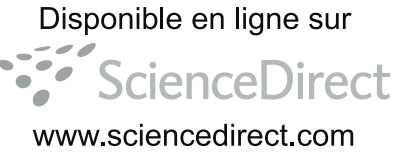

Diabetes \& Metabolism 40 (2014) S1-S3

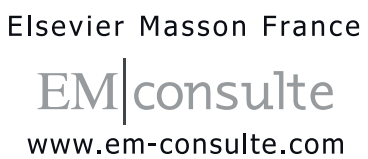

www.em-consulte.com

Editorial

\title{
SGLT2 receptor inhibitors: An opportunity to revise our therapeutic strategy for type 2 diabetes?
}

Type 2 diabetes is among the most important and prevalent chronic diseases, and any novel therapy that is able to address chronic hyperglycaemia is welcome as another tool for clinicians to help prevent the development of diabetic complications [1]. It is widely recommended that, if lifestyle interventions are inadequate, the first line of drug treatment is metformin, followed by a sulphonylurea, a glitazone, a dipeptidyl peptidase (DPP)-4 inhibitor or even a glucagonlike peptide (GLP)-1 analogue, before starting insulin [2]. However, every antidiabetic drug class has its own side-effects and limitations, including metformin [3]. Sulphonylureas, glitazones and insulin all cause weight gain, which can worsen insulin resistance. Sulphonylureas and insulin can also cause hypoglycaemia, while pioglitazone can induce oedema, heart failure and bone fractures [2]. These limitations of the classical glucose-lowering therapies have paved the way towards the development and success of incretin-based therapies for the management of hyperglycaemia in type 2 diabetes. However, beyond the potential safety issues, a major drawback of DPP-4 inhibitors (and, to a lesser extent, of GLP-1 analogues) is that a substantial proportion of diabetic patients will not respond sufficiently to these drugs and, thus, will fail to achieve glycated haemoglobin $\left(\mathrm{HbA}_{1 \mathrm{c}}\right)$ targets. Therefore, diabetologists clearly need novel therapies with higher rates of clinical response, while the complexity of type 2 diabetes probably also requires combined therapies in most cases.

The issue of an appropriate combination of antidiabetic drugs for type 2 diabetes is not so easily solved, as has already been emphasized by the American Diabetes Association/ European Association for the Study of Diabetes position statement [2]. Also, the choices offered to clinicians may become even more complicated, as health authorities and the European Medicines Agency (EMA) have approved the use of SGLT2 inhibitors for a wide range of indications, from a simple association with metformin to a triple oral therapy after failure of a metformin-sulphonylurea combination and even in association with insulin.

Sodium-glucose cotransporter type 2 (SGLT2) inhibitors are glucose-lowering agents with a particular glucoretic mechanism of action, which allows significant reductions of both hyperglycaemia and body weight, a unique dual effect among oral glucose-lowering agents. In addition, even though SGLT2 inhibitors do not directly target insulin secretion or insulin sensitivity (as do other classical glucose-lowering agents), they can indirectly improve both beta-cell function and insulin action because of their potential to reduce glucotoxicity [4].

The arrival of the SGLT2 inhibitors may be viewed as an exciting opportunity to review our concept of how best to treat type 2 diabetes. Indeed, because of their specific and insulin-independent mechanisms of action, SGLT2 inhibitors may be combined with any other type of antidiabetic therapy. This means that physicians will have the opportunity to test novel combinations of antidiabetic drugs targeting persistent hyperglycaemia, particularly in patients who do not respond adequately to the conventional first and second lines of treatment [5]. Even after failure of metformin monotherapy, it may be of benefit to add an SGLT2 inhibitor instead of a sulphonylurea to avoid hypoglycaemia, weight gain and subsequent deterioration of glucose control. At such a stage of the disease, an SGLT2 inhibitor (canagliflozin) has been shown to be more effective than a DPP-4 inhibitor (sitagliptin), while producing significant weight loss and blood pressure reduction. Also, after the failure of dual therapy with metformin and sulphonylurea, it may be tempting to introduce an SGLT2 inhibitor either in combination with those same two drugs or after substituting the sulphonylurea with the SGLT2 inhibitor. Such a combination might be proposed as a priority in overweight or obese patients without renal failure to avoid hypoglycaemia and postpone the introduction of insulin therapy.

SGLT2 inhibitors represent a valuable opportunity to shed new light on the association of antidiabetic drugs with sulphonylureas (with or without metformin). Beyond pioglitazone, which is not indicated for all patients and not available in all countries, the association of a sulphonylurea with a DPP-4 inhibitor is often of modest cumulative value. The additional decrease in $\mathrm{HbA}_{1 \mathrm{c}}$ conferred by such an association of two different insulin secretagogues, both targeting beta cells, is limited and less than $0.7 \%$ in most randomized clinical interventional studies, and the effect on glycaemia control 
weakens rapidly over time [6]. It would be more interesting and logical to use a combination of a sulphonylurea, which directly stimulates beta cells, with an SGLT2 inhibitor, which has a complementary mode of action independent of beta-cell responsiveness. The decrease in $\mathrm{HbA}_{1 c}$ conferred by such an association in published trial reports supports the use of this combination, as the reduction in $\mathrm{HbA}_{1 \mathrm{c}}$ vs placebo appears to be superior to that observed with a sulphonylurea plus a DPP-4 inhibitor, especially in patients with poor glucose control [5].

Of potential interest is the combination of an SGLT2 inhibitor with a sulphonylurea, which may be relevant for patients who cannot tolerate metformin [7]. Around 10-15\% of type 2 diabetes patients are intolerant $e_{\Omega}$ metformin, and the only oral combination available for such cases is the combination of a sulphonylurea with either pioglitazone (not available in all countries) or a DPP-4 inhibitor (not the most logical pharmacological combination, as discussed above).

One interesting dilemma for clinicians is having to choose between a DPP-4 inhibitor and an SGLT2 inhibitor after failure of metformin monotherapy or metformin plus sulphonylurea as combined therapy. Few head-to-head trials are available to allow an evidence-based answer to this question. However, superiority in reducing $\mathrm{HbA}_{1 \mathrm{c}}$ was demonstrated with a total 300-mg dose of canagliflozin compared with 100-mg sitagliptin in type 2 diabetes patients not well controlled with either metformin monotherapy [8] or metformin plus sulphonylurea [6], along with the added advantage of weight loss and blood pressure reduction.

Beyond the formal indications endorsed by the EMA and health authorities for SGLT2 inhibitors, there remains a wide field of clinical settings in which this class of drugs could be of interest to clinicians. Unfortunately, data on the efficacy of the association of SGLT2 inhibitors with DPP-4 inhibitors in dual or triple therapy remain scarce and have only been published as abstracts: empagliflozin plus linagliptin [9] and dapagliflozin plus saxagliptin [10]. Up till now, dual therapy with an SGLT2 inhibitor plus a DPP-4 inhibitor was not officially authorized for reimbursement in a number of countries. Yet, such an association is logical, as it combines two different but complementary mechanisms of action without inducing hypoglycaemia and weight gain.

Recent observations showing increased endogenous hepatic glucose production with SGLT2 inhibitors suggest the potential value of combining a drug that targets glucagon secretion (such as a DPP-4 inhibitor or GLP-1 analogue) with an SGLT2 inhibitor [4]. Another alternative would be to use an SGLT2 inhibitor in combination with metformin (or pioglitazone), which inhibits liver neoglucogenesis and contributes to a decrease of fasting glycaemia.

In addition, despite the lack of published data on the combination of GLP-1 analogues with SGLT2 inhibitors, this association could, in theory, induce the most weight loss, which might be particularly relevant for obese type 2 diabetes patients with poor glycaemic control [11].

Furthermore, the association of SGLT2 inhibitors with insulin, particularly for patients who gain considerable weight with insulin therapy and maintain high $\mathrm{HbA}_{1 \mathrm{c}}$ concentrations, appears to be logical. However, the results of randomized interventional trials assessing the efficacy of SGLT2 inhibitors plus insulin have been somewhat disappointing. The duration of these studies was limited, with rather modest decreases in $\mathrm{HbA}_{1 \mathrm{c}}$ compared with the control group ( $-0.5 \%$ to $\left.-0.6 \%\right)$. Nevertheless, in most of these studies, a substantial reduction in daily insulin dose was observed and significant weight loss was also reported [5]. Further large studies are now required to better delineate the appropriate place of, and clinical benefits conferred by, SGLT2 inhibitors given in addition to insulin. In the near future, SGLT2 inhibition might also be considered in conjunction with insulin therapy for type 1 diabetes [12].

Nevertheless, the use of SGLT2 inhibitors in diabetes patients with mild-to-moderate renal disease remains questionable. Due to their mechanism of action, the decrease in plasma glucose conferred by SGLT2 inhibitors is attenuated in the presence of chronic renal failure. However, preliminary reports suggest that the incidence of side-effects and overall safety profile are not substantially modified in the presence of mild renal disease [13]. In addition, consistent evidence has emphasized the importance of SGLT2 cotransporters in the pathophysiology of renal hyperfiltration [14,15]. Furthermore, the results of a few studies in rodents suggest amelioration of some of the features of diabetic nephropathy [16], although further clinical evidence is needed before this class of drugs can be recommended for patients with diabetic nephropathy. For this reason, the results of the currently ongoing, doubleblind, placebo-controlled CREDENCE (ClinicalTrials.gov Identifier: NCT02065791) and CANVAS-R (Study of the Effects of Canagliflozin on Renal Endpoints in Adult Subjects with T2DM; ClinicalTrials.gov Identifier: NCT01989754) trials, assessing whether the SGLT2 inhibitor canagliflozin can slow the progression of diabetic nephropathy, are eagerly awaited.

Beyond the effect of $\mathrm{HbA}_{1 \mathrm{c}}$ reduction per se, SGLT2 inhibitors have compared favourably with most other antidiabetic agents whatever the composite endpoints used in clinical trials, usually a combination of various items including the proportion of patients with $\mathrm{HbA}_{1 \mathrm{c}}<7 \%$, body weight changes, blood pressure reductions and/or rate of hypoglycaemic events [17]. Because SGLT2 inhibitors may have a favourable impact on various cardiovascular risk factors, a positive effect on cardiovascular outcomes is to be expected and, indeed, is currently being investigated in various prospective clinical trials using different SGLT2 inhibitors, including DECLARE (Dapagliflozin Effect on Cardiovascular Events)TIMI 58 (ClinicalTrials.gov Identifier: NCT01730534), CANVAS (Canagliflozin Cardiovascular Assessment Study; ClinicalTrials.gov Identifier: NCT01032629) and the EMPA-REG OUTCOME (Cardiovascular Outcome Trial of Empagliflozin; ClinicalTrials.gov Identifier: NCT01131676) [5].

In conclusion, the arrival of the SGLT2 inhibitors, a novel class of drugs with a unique insulin-independent mechanism of action, is a genuine opportunity to revise and re-imagine the 
possibilities for individualized therapies for type 2 diabetes patients. This is good news for diabetologists, provided that the long-term safety profile of this class of drugs proves demonstrably acceptable. Further additional randomized clinical studies are also warranted to specifically test novel combinations (such as a GLP-1 analogue with an SGLT2 inhibitor) in various clinical settings of type 2 diabetes.

\section{Disclosure of interest}

F. Bonnet has received honoraria from AstraZeneca/BMS, Boehringer Ingelheim, Eli Lilly, Janssen, Merck Sharp \& Dohme, Novartis, Novo Nordisk, Sanofi-Aventis and Takeda.

A.J. Scheen has received lecturer/advisor fees from Abbott, AstraZeneca/BMS, Boehringer Ingelheim, Eli Lilly, GlaxoSmithKline, Janssen, Merck Sharp \& Dohme, Novartis, Novo Nordisk, Sanofi-Aventis and Takeda.

\section{References}

[1] Scheen AJ, Charbonnel B. Effects of glucose-lowering agents on vascular outcomes in type 2 diabetes: A critical reappraisal. Diabetes Metab 2014;40:176-85.

[2] Inzucchi SE, Bergenstal RM, Buse JB, Diamant M, Ferrannini E, Nauck M, et al. Management of hyperglycaemia in type 2 diabetes: a patient-centered approach. Position statement of the American Diabetes Association (ADA) and the European Association for the Study of Diabetes (EASD). Diabetologia 2012;55:1577-96.

[3] Scheen AJ, Paquot N. Metformin revisited: A critical review of the benefit-risk balance in at-risk patients with type 2 diabetes. Diabetes Metab 2013;39:179-90.

[4] Scheen AJ, Paquot N. Metabolic effects SGLT2 inhibitors beyond increased glucosuria: a review of clinical evidence. Diabetes Metab 2014 (in press)

[5] Scheen AJ. Pharmacodynamics, efficacy and safety of SGLT2 Inhibitors for the treatment of type 2 diabetes. Drugs 2014;

[6] Schernthaner G, Gross JL, Rosenstock J, Guarisco M, Fu M, Yee $\mathrm{J}$, et al. Canagliflozin compared with sitagliptin for patients with type 2 diabetes who do not have adequate glycemic control with metformin plus sulfonylurea: a 52-week randomized trial. Diabetes Care 2013;36:2508-15.

[7] Strojek K, Yoon KH, Hruba V, Sugg J, Langkilde AM, Parikh S. Dapagliflozin added to glimepiride in patients with type 2 diabetes mellitus sustains glycemic control and weight loss over 48 weeks: a randomized, double-blind, parallel-group, placebo-controlled trial. Diabetes Ther 2014;5:267-83.
[8] Lavalle-Gonzalez FJ, Januszewicz A, Davidson J, Tong C, Qiu R, Canovatchel W, et al. Efficacy and safety of canagliflozin compared with placebo and sitagliptin in patients with type 2 diabetes on background metformin monotherapy: a randomised trial. Diabetologia 2013;56:2582-92.

[9] Patel S, DeFronzo R, Lewin A, Liu D, Kaste R, Woerle HJ, et al. Fixed dose combinations of empagliflozin/linagliptin for 52 weeks as add-on to metformin in subjects with type 2 diabetes. Diabetologia 2014;57(Suppl 1):S7, Abstract 1.

[10] Hansen L, Zee P, Li W, Cook B, Hishberg B, Iqbal N, et al. Randomised, double-blind trial of dual add-on saxagliptin plus dapagliflozin vs saxagliptin or dapagliflozin add-one alone in poorly controlled type 2 diabetes on metformin. Diabetologia 2014;57(Suppl 1):S8, abstract 4.

[11] Scheen AJ, Van Gaal LF. Combating the dual burden: therapeutic targeting of common pathways in obesity and type 2 diabetes. Lancet Diabetes Endocrinol 2014 (in press).

[12] Tahrani AA, Barnett AH, Bailey CJ. SGLT inhibitors in management of diabetes. Lancet Diabetes Endocrinol 2013;1:140-51.

[13] Gilbert R. SGLT-2 inhipitin in patients with kidney disease. Diabetes Metab 2014 (in press)

[14] Vallon V, Thomson SC. Kenal function in diabetic disease models: the tubular system in the pathophysiology of the diabetic kidney. Annu Rev Physiol 2012;74:351-75.

[15] Thomas M, Jandeleit-Dahm K, Bonnet F. Beyond Glycosuria: Exploring the intra-renal ef of SGLT 2 inhibition in diabetes. Diabetes Metab 2014 (in press).

[16] Terami N, Ogawa D, Tachibana H, Hatanaka T, Wada J, Nakatsuka A, et al. Long-term treatment with the sodium glucose cotransporter 2 inhibitor, dapagliflozin, ameliorates glucose homeostasis and diabetic nephropathy in db/db mice. PLoS One 2014;9:e100777.

[17] Einarson TR, Garg M, Kaur V, Hemels ME. Composite endpoints in trials of type-2 diabetes. Diabetes Obes Metab 2014;16:492-9.

Fabrice Bonnet ${ }^{\mathrm{a}}$, André J. Scheen ${ }^{\mathrm{b}}$ ${ }^{a}$ Service Endocrinologie-Diabétologie, CHU Rennes, Université Rennes 1, Inserm UMR 991, Rennes, France E-mail address: fabrice.bonnet@chu-rennes.fr

${ }^{b}$ University of Liège, Center for Interdisciplinary Research on Medicines (CIRM), Division of Diabetes, Nutrition and Metabolic Disorders and Division of Clinical Pharmacology, Department of Medicine, CHU Sart Tilman, University of Liège, Liège, Belgium

E-mail address: andre.scheen @ chu.ulg.ac.be 